\title{
314 - Effects of Transcranial Direct Current Stimulation (tDCS) on Cognitive Function in Alzheimer's Dementia
}

Carol Sheei-Meei Wang ${ }^{1,2,3 *}$, Kuo-Sheng Cheng ${ }^{2}$, Chia-Hung Tang ${ }^{1}$, Nien-Tsen Hou ${ }^{4}$, Pei-Fang Chien ${ }^{1}$, Ying-Che Huang ${ }^{4}$

${ }^{1}$ Department of Psychiatry, Tainan Hospital, Ministry of Health and Welfare, Tainan City, Taiwan ${ }^{2}$ Department of BioMedical Engineering, National Cheng Kung University, Tainan City, Taiwan ${ }^{3}$ Department of Psychiatry, National Cheng Kung University Hospital, Tainan City, Taiwan ${ }^{4}$ Department of Neurology, Tainan Hospital, Ministry of Health and Welfare, Tainan City, Taiwan

Introduction: Identifying effective treatments is a critical issue for Alzheimer's dementia (AD). The pathological amyloid deposits of $A D$ result in disruption of the balance between long-term potentiation (LTP) and long-term depression (LTD) of neuronal cells and synaptic plasticity. Brain stimulation in dementia research, especially with relatively safe tDCS, has been taken seriously recently. In theory, tDCS affects long-term synaptic plasticity through LTP and LTD, thereby improving cognitive ability. Recently, an increasing number of studies have been conducted to evaluate the efficacy of tDCS in AD and concluded a positive therapeutic effect. Currently, there are no studies of tDCS for AD in Taiwan. In this study, we investigate the effects of tDCS in AD.

Method: Using a double-blind, randomized and sham- controlled trial design, Sixteen AD aged 55-90 years ( 8 active, mean age 73.88 and 8 sham, mean age 74.75 ) were included in the study. AD diagnostics is according to DSM- 5 criteria. The CDR ratings of AD participants ranged from 0.5 to 2 . All subjects completed ten consecutive daily sessions in which they received either an active or a sham tDCS over the left dorsal lateral prefrontal cortex (anodal) and a cathodal electrode on the right supraorbital area. In each session, we applied a current intensity of $2 \mathrm{~mA}$ and an electrode size of $35 \mathrm{~cm}^{2}$ for 30 min in the active group. All subjects received a series of neuropsychological tests, which included CDR, MMSE, CASI and WCST, before and after these treatment sessions on the first day and 4 weeks later. Chi-square test, Wilcoxon signed ranks test and Mann-Whitney $U$ test were used to assess the differences in participant demographic characteristics and to compare the differences among groups.

Results: The active group showed significant improvement in total correct item, Conceptual level Responses (reflecting insight into the correct sorting principles), Categories Completed (reflecting overall success), and Trials to complete first categories (reflecting initial conceptual ability) of WCST 4 weeks later after the final stimulation. There were no statistically significant differences between before and after the 10-session course for the sham group.

Conclusion: tDCS stimulation improves cognitive operation and Conceptual Ability of AD.

Key words: Transcranial Direct Current Stimulation, Alzheimer's dementia, cognitive function 